\title{
Exosomes from adipose-derived stem cells overexpressing Nrf2 accelerate cutaneous wound healing by promoting vascularization in a diabetic foot ulcer rat model
}

Xue Li $\mathrm{Li}^{1,2}$, Xiaoyun Xie ${ }^{1,2}$, Weishuai Lian ${ }^{1,2}$, Rongfeng Shi, ${ }^{1,2}$, Shilong Han ${ }^{1,2}$, Haijun Zhang ${ }^{2,3}$, Ligong Lu ${ }^{2,4}$ and Maoquan Li $\mathbb{B}^{1,2}$

\begin{abstract}
Diabetic foot ulcers (DFU) increase the risks of infection and amputation in patients with diabetes mellitus (DM). The impaired function and senescence of endothelial progenitor cells (EPCS) and high glucose-induced ROS likely exacerbate DFUs. We assessed EPCs in 60 patients with DM in a hospital or primary care setting. We also evaluated the therapeutic effects of exosomes secreted from adipose-derived stem cells (ADSCs) on stress-mediated senescence of EPCs induced by high glucose. Additionally, the effects of exosomes and Nrf2 overexpression in ADSCs were investigated in vitro and in vivo in a diabetic rat model. We found that ADSCs that secreted exosomes promoted proliferation and angiopoiesis in EPCs in a high glucose environment and that overexpression of Nrf2 increased this protective effect. Wounds in the feet of diabetic rats had a significantly reduced ulcerated area when treated with exosomes from ADSCs overexpressing Nrf2. Increased granulation tissue formation, angiogenesis, and levels of growth factor expression as well as reduced levels of inflammation and oxidative stress-related proteins were detected in wound beds. Our data suggest that exosomes from ADSCs can potentially promote wound healing, particularly when overexpressing Nrf2 and therefore that the transplantation of exosomes may be suitable for clinical application in the treatment of DFUs.
\end{abstract}

\section{Introduction}

Diabetic foot ulcers (DFU) are accompanied by a major risk of infection and amputation in patients with diabetes mellitus $(\mathrm{DM})^{1,2}$ and can increase the risk of 5 -year mortality to $80 \%, 3$. Alterations in protein and lipid metabolism combined with peripheral neuropathy in DM

\footnotetext{
Correspondence: Haijun Zhang (ceo@brandentech.com) or Ligong Lu

(lulg@21cn.com) or Maoquan Li (cjr.limaoquan@vip.163.com)

'Department of Interventional \& Vascular Surgery, Tenth People's Hospital of

Tongji University, No. 301 Middle Yan Chang Road, Shanghai 200072, China

${ }^{2}$ Institute of Interventional \& Vascular Surgery, Tongji University, No. 301 Middle

Yan Chang Road, Shanghai 200072, China

Full list of author information is available at the end of the article
}

impede the normal process of wound healing by delaying the formation of mature granulation tissue with a simultaneous reduction in wound tensile strength $^{4-7}$. Hyperglycemia-induced metabolic abnormalities increase the activity of the enzymes aldose reductase and sorbitol dehydrogenase to convert intracellular glucose to sorbitol and fructose. An accumulation of these sugar products restricts the synthesis of nerve cell myoinositol to prevent nerve conduction and reduces levels of nicotinamide adenine dinucleotide phosphate used in the detoxification of reactive oxygen species (ROS) and the synthesis of nitric oxide ${ }^{6}$. This combination of events results in neuropathy that causes anatomic foot deformities and

\section{(c) The Author(s) 2018}

Open Access This article is licensed under a Creative Commons Attribution-NonCommercial-NoDerivatives 4.0 International License, which permits any non-commercial (c) use, sharing, distribution and reproduction in any medium or format, as long as you give appropriate credit to the original author(s) and the source, and provide a link to the Creative Commons license. You do not have permission under this license to share adapted material derived from this article or parts of it. The images or other third party material in this article are included in the article's Creative Commons license, unless indicated otherwise in a credit line to the material. If material is not included in the article's Creative Commons license and your intended use is not permitted by statutory regulation or exceeds the permitted use, you will need to obtain permission directly from the copyright holder. To view a copy of this license, http://creativecommons.org/licenses/by-nc-nd/4.0/. 
consequently the breakdown of skin leading to skin ulceration ${ }^{8}$.

Hyperglycemia is associated with endothelial dysfunction and reduced blood vessel growth; therefore, angiogenesis is often compromised in patients with diabetes ${ }^{9}$. In angiogenesis, proteolysis of the extracellular matrix occurs through proteolytic systems such as matrix metalloproteinases. Endothelial cells then migrate toward the angiogenic stimulus at which they proliferate to form tubular structures and blood vessels. Insufficient angiogenesis and impaired wound healing occur when inflammatory cells are limited and the supply of oxygen and nutrients in the wound space is poor. A direct association is found between poor angiogenesis and high glucose levels ${ }^{10}$. Clinical trials show that the senescence of EPCs is promoted and their function impaired under high glucose-induced ROS and inflammation conditions ${ }^{11}$. Furthermore, senescence marker protein 30 (SMP30) is down regulated in patients with diabetic nephropathy ${ }^{12}$. SMP30 is a $34 \mathrm{kDa}$ cytosolic aging marker protein, also known as regucalcin, which is involved in intracellular $\mathrm{Ca}^{2}$

${ }^{+}$homeostasis, ascorbic acid biosynthesis, and the regulation of oxidative stress ${ }^{13}$. Consequently, a deficiency of SMP30 exacerbates oxidative stress and impairs angiogenic activity ${ }^{14}$.

The transcription factor nuclear factor-E2-related factor 2 (Nrf2) has a protective role against oxidative stress and has demonstrated potential therapeutic effects in a diabetic nephropathy animal model ${ }^{15}$. Under normal conditions, Nrf2 associates with Kelch-like ECH-associated protein 1 (KEAP1) in the cytoplasm and remains inactive. On exposure to oxidative stress, Nrf2 is released by KEAP and translocates into the nucleus and then regulates expression of antioxidant response element (ARE)-mediated expression of antioxidant enzymes and the cystine/ glutamate transporter involved in glutathione biosynthesis $^{16,17}$. Loss of Nrf2/ARE activity increases oxidative stress, which can aggravate the endothelial dysfunction and abnormal angiogenesis that occurs in diabetes ${ }^{18}$. Furthermore, patients with Nrf2 genetic variants are more susceptible to complications in diabetes, including peripheral neuropathy, nephropathy, retinopathy, foot ulcers, and microangiopathy ${ }^{19}$.

Adipose-derived stem cells (ADSCs) have the capacity to mediate wound healing through mitochondrial transfer and the secretion of paracrine factors and exosomes ${ }^{20-22}$. Furthermore, exosomes from ADSCs are internalized by fibroblasts to stimulate cell migration, proliferation, and collagen synthesis in a dose-dependent manner to significantly accelerate cutaneous wound healing ${ }^{23}$. In this work, we evaluated the therapeutic effect of exosomes secreted by ADSCs overexpressing Nrf2 on the high glucose-induced senescence of EPCs in a diabetic rat model of DFUs.
Table 1 Comparison between demographic and laboratory characteristics of diabetic patients and controls

\begin{tabular}{lll}
\hline Clinical parameter & Control & Diabetic \\
\hline No. of patients & 30 & 60 \\
Age (years) & $52.39 \pm 17.4$ & $57.74 \pm 13.32$ \\
Sex (male/female) & $17 / 13$ & $31 / 29$ \\
Body mass index (kg/m $\left.{ }^{2}\right)$ & $22.98 \pm 2.40$ & $25.88 \pm 4.55$ \\
Duration of diabetes (years) & 0 & $9.96 \pm 8.55$ \\
HbA1c (\%) & $5.2 \pm 0.43$ & $7.88 \pm 2.13^{* * *}$ \\
FPG (mg/dl) & $4.6 \pm 0.67$ & $7.44 \pm 1.38^{* * *}$ \\
TC (mg/dl) & $4.33 \pm 0.66$ & $4.46 \pm 0.96$ \\
TG (mg/dl) & $1.96 \pm 0.64$ & $2.27 \pm 1.3$ \\
DBP (mmHg) & $108.24 \pm 15.07$ & $115.42 \pm 16.62$ \\
CRP (mg/l) & $3.56 \pm 1.37$ & $4.05 \pm 1.58$ \\
VEGF (pg/ml) & $63.27 \pm 9.28$ & $124.22 \pm 22.33^{* * *}$ \\
SMP30 (pg/ml) & $107.24 \pm 19.33$ & $42.51 \pm 7.52^{* * *}$ \\
IL-6 (pg/ml) & $28.21 \pm 3.24$ & $58.24 \pm 3.35^{* * *}$ \\
IL-1 $3(\mathrm{pg} / \mathrm{ml})$ & $58.28 \pm 15.17$ & $125.37 \pm 31.52^{* * *}$ \\
TNF-a (pg/ml) & $25.22 \pm 17.28$ & $67.68 \pm 8.32^{* * *}$ \\
\hline
\end{tabular}

HbA1C whole blood glycated hemoglobin FPG fasting plasma glucose, $T C$ total cholesterol, $T G$ triglycerides, $D B P$ diastolic blood pressure, $C R P$ plasma C-reactive protein, VEGF vascular endothelial growth factor, SMP3O senescence marker protein-30.

Data are expressed as mean \pm SD. ${ }^{* *} P<0.01,{ }^{* * *} P<0.001$ against the control values

\section{Materials and methods \\ Reagents}

Antibodies against KDR, CD29, CD133, vWF, CD14, CD34, GAPDH, NOX1, NOX4, VEGFR2, pVEGFR2, SMP30, VEGF, Nrf2, and 2',7'-dichlorofluorescein diacetate (DCF-DA) were obtained from Sigma-Aldrich (St. Louis, MO, USA). Dulbecco's modified eagle medium (DMEM, high glucose), serum-free endothelial basal medium (EBM), and fetal bovine serum were from Nego (Shanghai, China). Cell lysis buffer $(10 \times)$ was obtained from Cell Signaling Technology (Danvers, MA, USA). The RT-PCR and ELISA kits were purchased from TOYOBO (Shanghai, China). Other reagents included DAPI (Roche, Basel, Switzerland), hematoxylin and eosin (H\&E; Toronto Chemicals, Toronto, Canada), trypsin and Masson trichrome (Sigma-Aldrich). Shenggong Biotechnology (Shanghai, China) synthesized all pairs of real-time PCR primers. Other chemicals and reagents were of analytical grade.

\section{Clinical specimen collection and ethics statement}

Peripheral blood samples were obtained from 60 patients with DM and from 30 healthy volunteers 
(Table 1). A standard format elaborated by the principal investigator with clinical features was included for age, sex, body mass index and relative growth factors and inflammatory factors. Samples were obtained from all 90 subjects. All specimens were frozen in liquid nitrogen immediately. Permission to use the samples for research purposes was obtained and approved by the Ethics Committee of the Shanghai Tenth People's Hospital of China, China, and a written consent form was obtained from all patients.

\section{Isolation and characterization of EPCs}

EPCs were cultured from peripheral blood, as described in previous reports ${ }^{24,25}$. Peripheral blood taken from patients was diluted in PBS $(2 \times)$ and layered over $4 \mathrm{ml}$ of lymphocyte separation liquid (Sigma-Aldrich) for centrifugation $\left(800 \times g, 30 \mathrm{~min}\right.$ at $\left.4{ }^{\circ} \mathrm{C}\right)$. The periphery blood mononuclear cells (PBMCs) in the interface layer were transferred to a new tube and washed with PBS buffer by centrifugation $\left(400 \times g\right.$ for $5 \mathrm{~min}$ at $\left.4{ }^{\circ} \mathrm{C}\right)$. EPCs were then cultured from PBMCs in endothelial cell basal medium-2 (Lonza, Walkersville, MD, USA) on a 24-well plate $(5 \times$ $10^{6}$ cells/well). Cells were cultured continuously for 10 days for coculture studies. EPCs were then selected using CD133-coupled magnetic microbeads (Miltenyi Biotech, Bergisch Gladbach, Germany) following the manufacturer's instructions. After isolation, CD133+ cells were expanded in DMEM medium. Immunophenotypic analysis was performed staining $5 \times 10^{5}$ isolated and expanded EPCs. The cells were incubated with conjugated monoclonal antibodies against CD34, KDR, CD133, and CD14 (Sigma-Aldrich). Isotype-identical antibodies were the controls (PharMingen). For analysis of CD34, KDR, CD133 and CD14, the cells were further incubated with a horse biotinylated anti-rat IgG (H1L) antibody (Vector Laboratories) and with FITC-conjugated streptavidin (Caltag, San Francisco, CA, USA). After treatment, the cells were fixed in 1\% paraformaldehyde. Quantitative analyses were performed using a FACSCalibur flow cytometer and FlowJo software (Flowjo, Ashland, OR, USA).

\section{ELISA for soluble inflammatory cytokines}

IL-6, IL-1 $\beta$, and TNF- $\alpha$ in the supernatants of EPCs or sera from subjects with/without diabetes or from mice with/without exosome treatment were measured using commercially available ELISA kits (Sen-Xiong Company). In accordance with the manufacturer's instructions, supernatants were stored at $-80^{\circ} \mathrm{C}$ before measurement, and both standards and samples were run in triplicate. $\mathrm{OD}_{450}$ was calculated by subtracting the background, and standard curves were plotted.

\section{Evaluation of oxidative stress levels}

ROS production in EPCs and skin tissue samples was measured using DCF-DA. EPCs $\left(1 \times 10^{6}\right.$ cells $)$ were seeded in $100 \mathrm{~mm}$ culture dishes and pretreated with DAPI for 30-60 min. Cells were then incubated with DCF-DA $(20 \mathrm{mM})$ for $10 \mathrm{~min}$ at $37^{\circ} \mathrm{C}$ in the dark. After a 10 -min incubation under hypoxia or normoxia conditions, ROS production in cells was assessed using an ECLIPSE E600 fluorescence microscope (Nikon). Additionally, the fluorescence intensity of DCF-DA was measured and calculated using flow cytometry (BD Biosciences, San Jose, CA, USA). ROS production was quantified in vivo immediately after removal of skin tissue from mice.

\section{Immunohistochemistry and immunofluorescence}

Skin tissue samples were fixed in $10 \%$ formalin solution and embedded in paraffin. Sections, $5 \mu \mathrm{m}$, were stained with $H \& E$ and Masson's trichrome. CD31 staining was performed to evaluate histopathological changes in angiogenesis. Sections were examined using an Axiophot light microscope (Zeiss, Oberkochen, Germany) or a fluorescence microscope (Nikon, Tokyo, Japan) and photographed with a digital camera.

\section{Flow cytometry}

Flow cytometry was used to determine the rate of apoptosis of EPCs. Apoptotic cells were differentiated from viable or necrotic cells by the combined application of annexin-V (AV)-FITC and propidium iodide (PI). Cells were washed twice and adjusted to a concentration of $1 \times$ $10^{6}$ cells $/ \mathrm{ml}$ with cold D-Hanks buffer. Then, AV-FITC $(10 \mu \mathrm{l})$ and PI $(10 \mu \mathrm{l})$ were added to $100 \mu \mathrm{l}$ of cell suspension and incubated for $15 \mathrm{~min}$ at room temperature in the dark. Finally, $400 \mu \mathrm{l}$ of binding buffer was added to each sample without washing and analyzed using flow cytometry. Each experiment was performed in triplicate.

\section{Tubule formation assay}

In vitro neovascularization assays were performed in human fibrin matrices. In brief, EPCs from different treatments were serum-starved and seeded onto a Matrigel-coated plate (BD Bioscience) in EBM medium and incubated at $37^{\circ} \mathrm{C}$ for $12 \mathrm{~h}$. Tubular structures of EPCs in the Matrigel were analyzed by phase-contrast microscopy. To quantify the length of newly formed tubes, six random phase-contrast photomicrographs were taken per well.

\section{Coculture of EPCs and ADSCs}

ADSCs were prepared as described in supplementary information. EPCs and ADSCs were cocultured using a Transwell system with a $0.4-\mu \mathrm{m}$ porous membrane (Costar, Corning, NY, USA) as previously described ${ }^{26}$. The EPCs were first planted and grown to an appropriate 
density in the lower wells, and then ADSCs were planted in the upper wells. Before ADSCs were cocultured with EPCs, they were pretreated with $2.5 \mu$ M GW4869 (SigmaAldrich) for $8 \mathrm{~h}$ to reduce the release of exosomes. All cells were cultured under high glucose $(30 \mathrm{mM}$, glucose) or normal (5.5 mM, glucose) conditions.

\section{Exosome labeling and uptake}

Exosomes were labeled with a Dil fluorescent labeling kit (Sigma-Aldrich). Dil $(400 \mu \mathrm{l})$ was added to the exosome suspension and incubated for $5 \mathrm{~min}$ at room temperature. The reaction was stopped by the addition of an equal volume of exosome-depleted bovine serum albumin, and then the exosomes were washed twice with PBS to remove any unbound dye. Subsequently, the Dillabeled or denatured exosomes were incubated with EPCs for $24 \mathrm{~h}$. The cells were then fixed and stained with DAPI. Images were obtained on a confocal microscope.

\section{Isolation and identification of human ADSC-derived exosomes}

At $80-90 \%$ confluence, ADSCs were rinsed with PBS and cultured in EGM-2MV media deprived of FBS and then supplemented with $1 \times$ serum replacement solution (PeproTech, Rocky Hill, NJ, USA) for an additional $24 \mathrm{~h}$. ADSCs in conditioned media were centrifuged at $300 \times g$ for $10 \mathrm{~min}$ and $2000 \times g$ for $10 \mathrm{~min}$ to remove dead cells and cellular debris. Then, $\sim 10 \mathrm{ml}$ of supernatant was mixed with $5 \mathrm{ml}$ of ExoQuick Exosome Precipitation Solution (SBI, CA, USA) and incubated at $4{ }^{\circ} \mathrm{C}$ for $12 \mathrm{~h}$. The ExoQuick/supernatant mixture was centrifuged at $1500 \times g$ for $30 \mathrm{~min}$ to obtain a pellet containing exosomes, which was then resuspended in $250 \mu \mathrm{l}$ of nucleasefree water. Total RNA and protein from exosomes were extracted using TRIzol-LS (Invitrogen, Carlsbad, CA, USA) and an Exosomal Protein Extraction kit (Invitrogen), following the manufacturer's instructions, respectively. Exosomes were stored at $-80^{\circ} \mathrm{C}$ or used for downstream experiments. Transmission electron microscopy (TEM) and western blotting were used to identify the collected exosomes. The size of vesicles was determined by a dynamic light scattering technique using a Zetasizer Nano ZS analysis system (Zetasizer version 6.12; Malvern Instruments, UK). The $x$-axis on a size distribution plot shows the estimated distribution of particle radii $(\mathrm{nm})$, with the $y$-axis showing the relative percentages.

\section{Diabetic skin wound animal model and treatment}

Adult female Sprague Dawley rats (4-6 weeks, 150-200 g, grade: clean, license: SCXK 2013-0005) were purchased from SLAC Laboratory Animal Co., Ltd (Shanghai, China) and used to establish a rat model for diabetes as described previously ${ }^{27}$. All animal experiments were conducted in accordance with the National Institutes of Health Guide for the Care and Use of Laboratory Animals and approved by the Biological Research Ethics Committee of the Chinese Academy of Sciences. Rats were maintained on a 12 $\mathrm{h}$ light cycle in the animal facility of the Shanghai Tenth People's Hospital of China. Streptozotocin was injected intraperitoneally $(100 \mathrm{mg} / \mathrm{kg}$ in $0.01 \mathrm{M}$ sodium citrate, $\mathrm{pH}$ 4.3) to induce diabetes. Blood glucose level was maintained between 16.7 and $33.3 \mathrm{mmol} / \mathrm{l}$ by $6-18$ units/day of insulin (Wan-Bang Biochemical Medicine Co. Ltd, Xuzhou, China). After 4 weeks, a model of DFUs was created in rats anesthetized with isoflurane inhalation. A single round full-thickness skin wound was created with a 5-mm disposable skin biopsy punch and Westcott scissors on the dorsum of hind feet of the diabetic rats.

\section{Measurement of ulceration contraction rate}

Before harvesting, the size of the wounded area on each rat's foot was measured with a ruler and recorded on parfocal digital photographs taken at Day 0, 7, and 15 after injecting PBS as a control or EPCs, EPCs combined with exosomes from ADSCs or Nrf2 overexpression ADSCs. Images were taken with a digital camera (DMC-LX5GK; Panasonic, Japan), and the ulcerated area was analyzed by Image-Pro Plus 4.5 software.

\section{Western blot analysis}

Western blot analysis was conducted using cell lysates in urea buffer ( $8 \mathrm{M}$ urea, $1 \mathrm{M}$ thiourea, 0.5\% CHAPS, 50 $\mathrm{mM}$ dithiothreitol, $24 \mathrm{mM}$ spermine). Cytoplasmic and nuclear protein fractions were prepared using either NEPER nuclear or cytoplasmic extraction reagents (Pierce, Rockford, IL, USA), respectively, following manufacturer's protocols. $\beta$-tubulin was used as a loading control for the cytoplasmic fraction, whereas GAPDH was used as a loading control. Samples $(40 \mu \mathrm{g}$ of total protein) were separated on SDS-PAGE and transferred to nitrocellulose membranes (Millipore, Jaffrey, NH, USA). After blocking in $5 \%$ nonfat milk for $1 \mathrm{~h}$, the membranes were incubated with primary antibodies against SMP30 (1:1000), VEGF (1:200), p-VEGFR2 (1:200), VEGFR2 (1:200), ASPP2 (1:400), E-cadherin (1:300), N-cadherin (1:400), GSK3 $\beta$ (1:200), NOX1 (1:200), NOX4 (1:500), CD63 (1:400), TSC101 (1:400), CD9 (1:200), $\beta$-actin (1:200), and GAPDH (1:2000) at $4{ }^{\circ} \mathrm{C}$ overnight. After washing, the membranes were incubated with horseradish peroxidaseconjugated secondary antibodies for $1 \mathrm{~h}$ at room temperature. Signals were detected using an ECL detection system (GE Healthcare, Aurora, OH, USA) and analyzed by ImageJ 1.42q software (National Institutes of Health).

\section{Transmission electron microscopy}

TEM was used to identify the morphology of exosomes. Briefly, ADSC exosomes were fixed in 3\% glutaraldehyde 


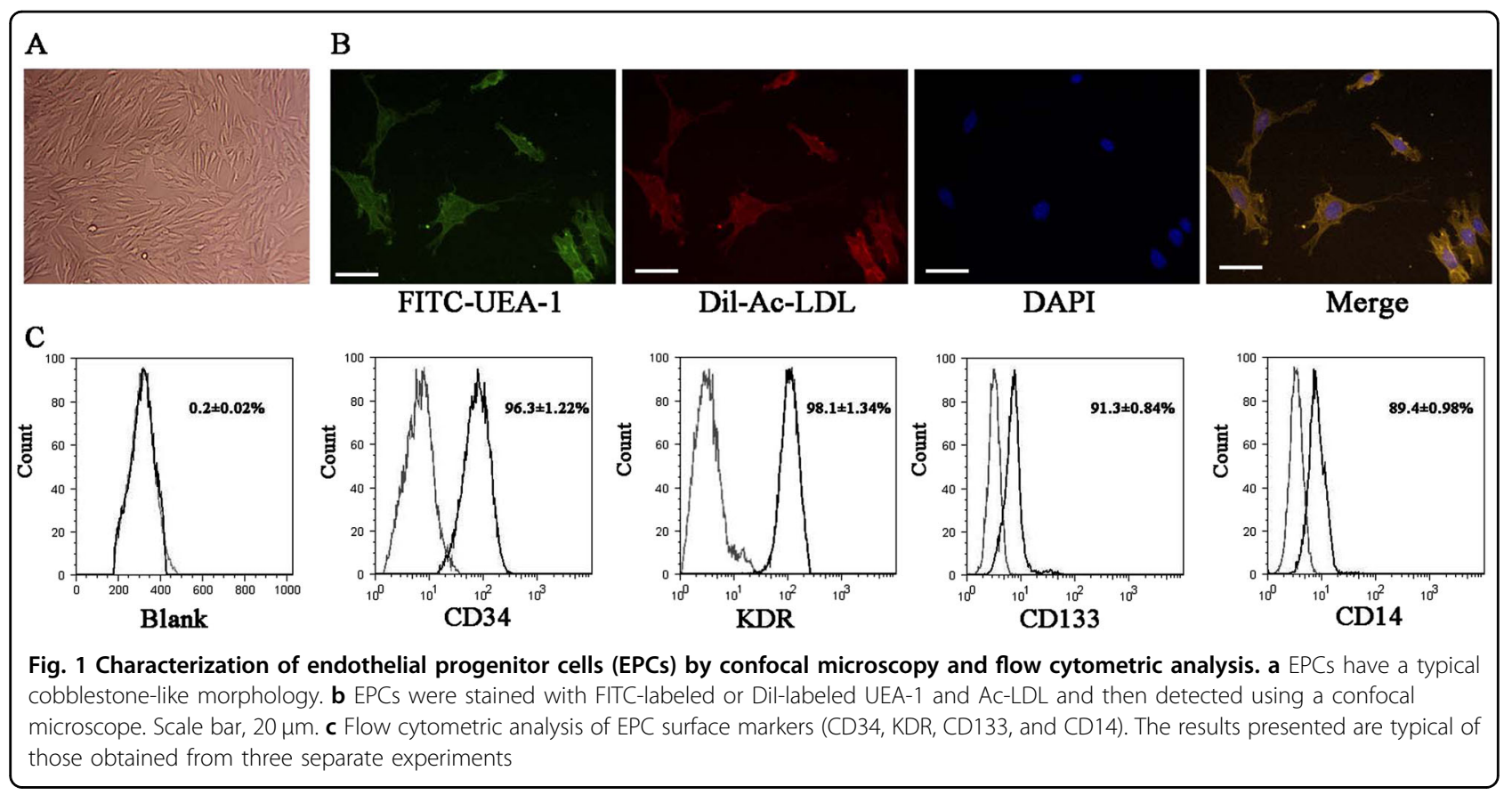

for $2 \mathrm{~h}$ and washed with PBS two times. The exosomes were negatively stained with $2 \%$ uranyl acetate for $30 \mathrm{sec}$ and applied to a continuous carbon grid. Exosomes were visualized on a Hitachi H-7650 TEM (Hitachi, Tokyo, Japan), and images were captured using a digital camera (Olympus, Tokyo, Japan).

\section{Statistical analyses}

Results are expressed as the mean \pm standard deviation (SD). Statistical significance was evaluated by analysis of variance followed by Tukey-Kramer multiple comparisons test and by Student's $t$-test. $P<0.05$ denotes statistical significance.

\section{Results}

\section{High glucose accelerates stress-induced premature senescence in EPCs}

Demographic and laboratory characteristics of patients with diabetes were compared to subjects without diabetes (Table 1). In patients with diabetes, levels of fasting glucose, whole blood glycated hemoglobin, vascular endothelial growth factor (VEGF), and the inflammatory factors interleukin (IL)-6, IL-1 $\beta$, and tumor necrosis factor alpha (TNF- $\alpha$ ) were significantly elevated. By contrast, levels of SMP30 were significantly reduced in patients with diabetes $(P<0.001)$. EPCs were cultured from the peripheral blood of patients with diabetes and confirmed by confocal microscopy (Fig. 1a,b) and flow cytometry using EPC surface markers (CD34, KDR, CD133, and CD14; Fig. 1c).

SMP30 and VEGFR2 were further assessed, in addition to the oxidative stress-related proteins NOX1 and NOX4, in circulating CD34-positive cells of DM patients and healthy controls. In western blot assessments, the levels of SMP30 and VEGFR2 were reduced in DM patients $(P<$ 0.001 vs. control group; Fig. 2a,b), whereas those of NOX1 and NOX4 increased significantly $(P<0.001$ vs. control group; Fig. 2c). These results indicate that senescence and oxidative stress increased in patients with DM. The tube formation capability of EPCs in DM patients was measured using a Matrigel-based assay, and results indicate that the tube formation capability of EPCs from patients with DM decreased (Fig. 2d).

EPCs from subjects without DM were incubated with increasing concentrations of glucose for 24-72 h, and cell viability was measured by MTT assay (Fig. 3a). High glucose levels $(30 \mathrm{mM})$ significantly reduced cell viability compared with that in normal glucose $(5.5 \mathrm{mM})$ after 48 $(P<0.05)$ and $72 \mathrm{~h}(P<0.01)$. The intracellular ROS level of EPCs was determined by immunofluorescence using DCH-DA staining after exposure to high glucose for different lengths of time. Immunofluorescence images and superoxide anion measurements of fluorescent intensity with dihydroethidium (DHE) indicated gradually increasing levels of ROS, which peaked at $72 \mathrm{~h}$ (Fig. 3b,c) $(P<0.001$ vs. normal glucose). Western blot analysis was used to measure protein expression levels of SMP30, VEGF, VEGFR2, and NOX1/4 under high glucose conditions. Levels of SMP30 and VEGFR2 phosphorylation significantly decreased, whereas levels of VEGF, NOX1, and NOX4 significantly increased, indicating an increase in senescence and levels of ROS (Fig. 3d). Relative protein levels are shown in Fig. $3 \mathrm{e}-\mathrm{i}$. Apoptosis determined by 


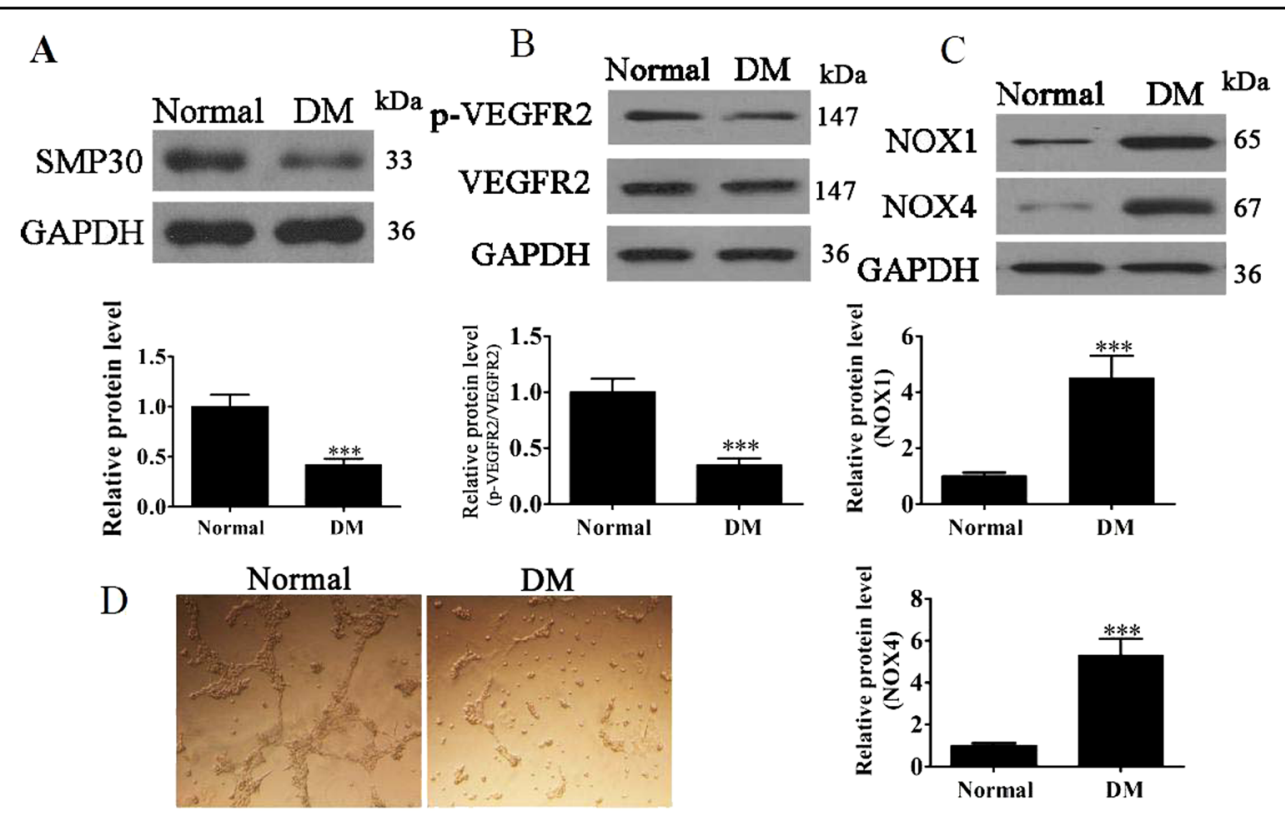

Fig. 2 Expression of SMP30 and oxidative stress-related proteins on circulating CD34-positive cells of patients with diabetes mellitus (DM) and healthy controls. a Expression of senescence marker protein 30 (SMP30) was measured by western blot. GAPDH served as an internal control. The relative protein levels were analyzed, and data are presented as the mean \pm SD $(n=3)$. ${ }^{* *} P<0.001$ vs. normal group. b Phosphorylation of VEGFR2 was measured by western blot. GAPDH served as an internal control. The relative protein levels were analyzed, and data are presented as the mean \pm SD $(n=3) .{ }^{* *} P<0.001$ vs. normal group. $\mathbf{c}$ Levels of the ROS-related proteins NOX1 and NOX4 were measured by western blot. GAPDH served as an internal control. The relative protein levels were analyzed, and data are presented as the mean \pm SD $(n=3)$. ${ }^{* * *} P<0.001$ vs. normal group. $\mathbf{d}$ The tube formation capability of endothelial progenitor cells (EPCs) from DM patients or subjects without DM was measured. The results show that the tube formation capability of EPCs decreased in DM patients

Annexin-V/PI staining confirmed an increase in senescence in EPCs under a condition of high glucose (Fig. 3j). Inflammatory cytokine levels (IL-1 $\beta$, IL-6, and TNF- $\alpha$ ) measured with ELISA were also significantly higher in EPCs grown under high glucose conditions (Fig. 3k-m) than those under normal glucose.

Overall, these results confirm that hyperglycemia promotes the premature senescence of EPCs and increases levels of ROS and inflammation.

\section{Exosomes derived from ADSCs reduce glucose-induced EPC senescence}

We determined the glucose-induced apoptosis rate of EPCs cocultured with ADSCs with or without GW4869, a neutral sphingomyelinase inhibitor that blocks exosome generation, by Annexin-V/PI staining. The method for preparing ADSCs can be found in supplementary information (Supplemental S1). The percentage of apoptotic cells was significantly reduced when EPCs were cocultured with ADSCs, but no difference in apoptotic rate was observed with the addition of GW4869 (Fig. 4a,b). Moreover, tube formation capability increased in EPCs stimulated with ADSCs, but these effects decreased when ADSCs were pretreated with GW4869 (Fig. 4c). The observations were similar for intracellular ROS levels determined by immunofluorescence using DCH-DA stain and measuring the fluorescence intensity of DHE (Fig. 4d, e). The presence of ADSCs reduced glucose-induced ROS levels in EPCs, but these benefits were reversed by the addition of the exosome blocker GW4869. Protein expression levels of SMP30, VEGF, VEGFR2, NOX1, and NOX4 were measured by western blot (Fig. 4f), and relative protein levels are shown in Fig. 4g-k. Levels of SMP30 and VEGF were significantly reduced in EPCs cocultured with ADSCs but not when ADSCs were pretreated with GW4869. By contrast, levels of VEGFR2 phosphorylation, NOX1 and NOX4 were reduced in the presence of ADSCs, but no difference was observed when ADSCs were pretreated with GW4869. Similarly, inflammatory cytokine levels of IL- $1 \beta$, IL- 6 , and TNF- $\alpha$ measured with ELISA in EPCs were reduced significantly by the presence of ADSCs $(P<0.001)$, but no difference was observed when ADSCs were pretreated with GW4869 (Fig. 4l-n).

TEM was used to isolate and identify exosomes from the ADSC culture medium. Figure $5 \mathrm{a}$ shows the ultrastructure of ADSC-derived exosomes with a diameter of approximately $100 \mathrm{~nm}$. The size distribution was slightly 


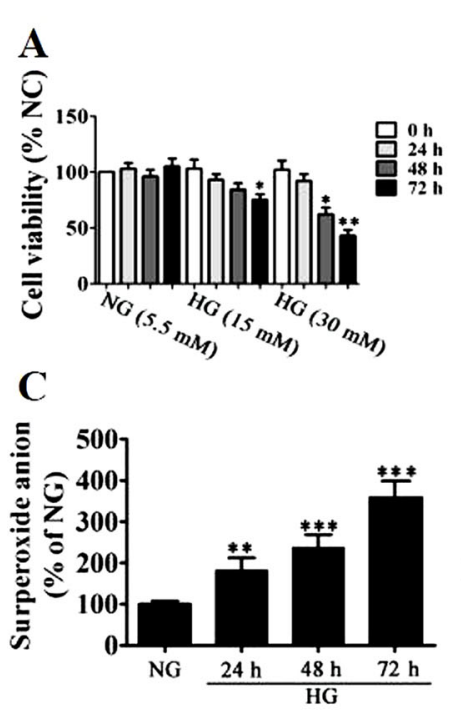

B

D
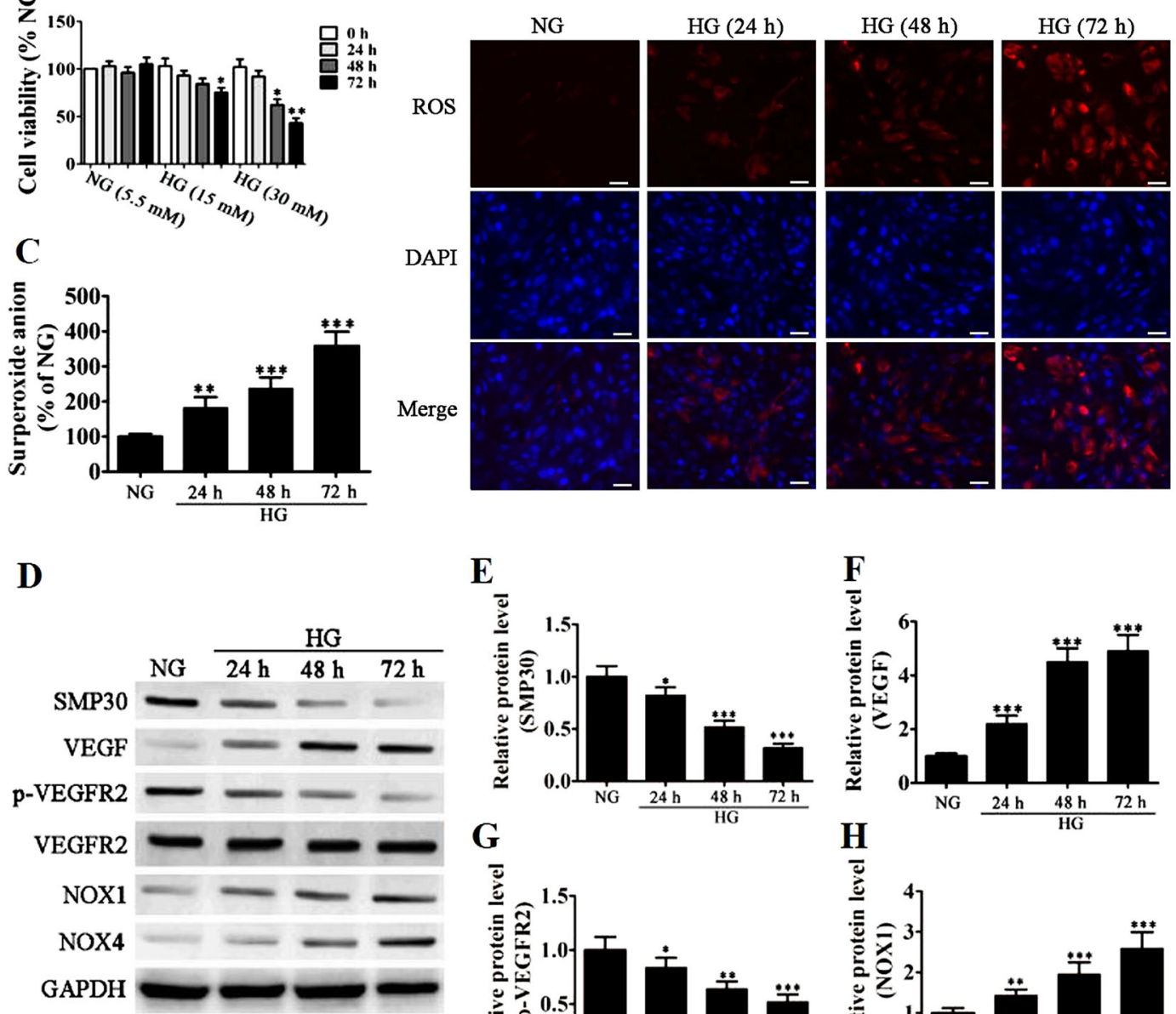

E
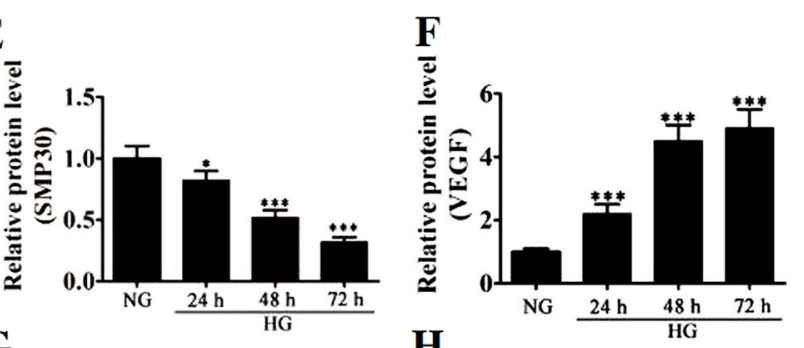

G
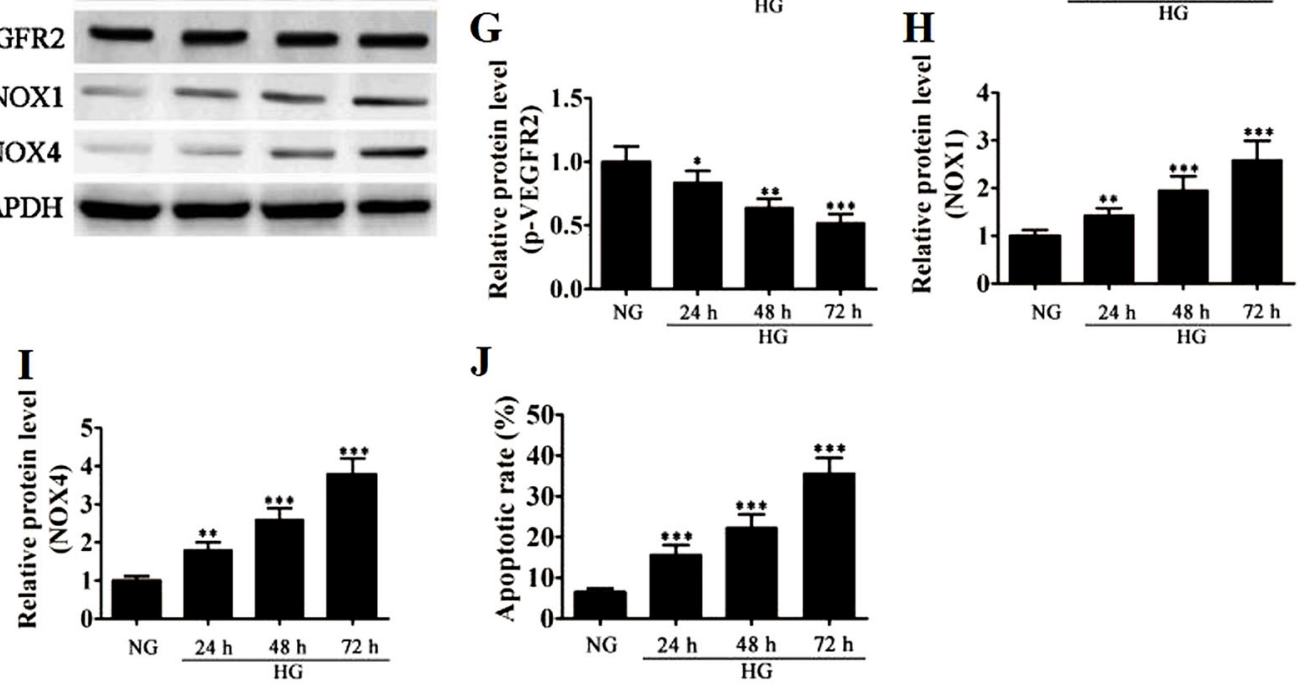

J
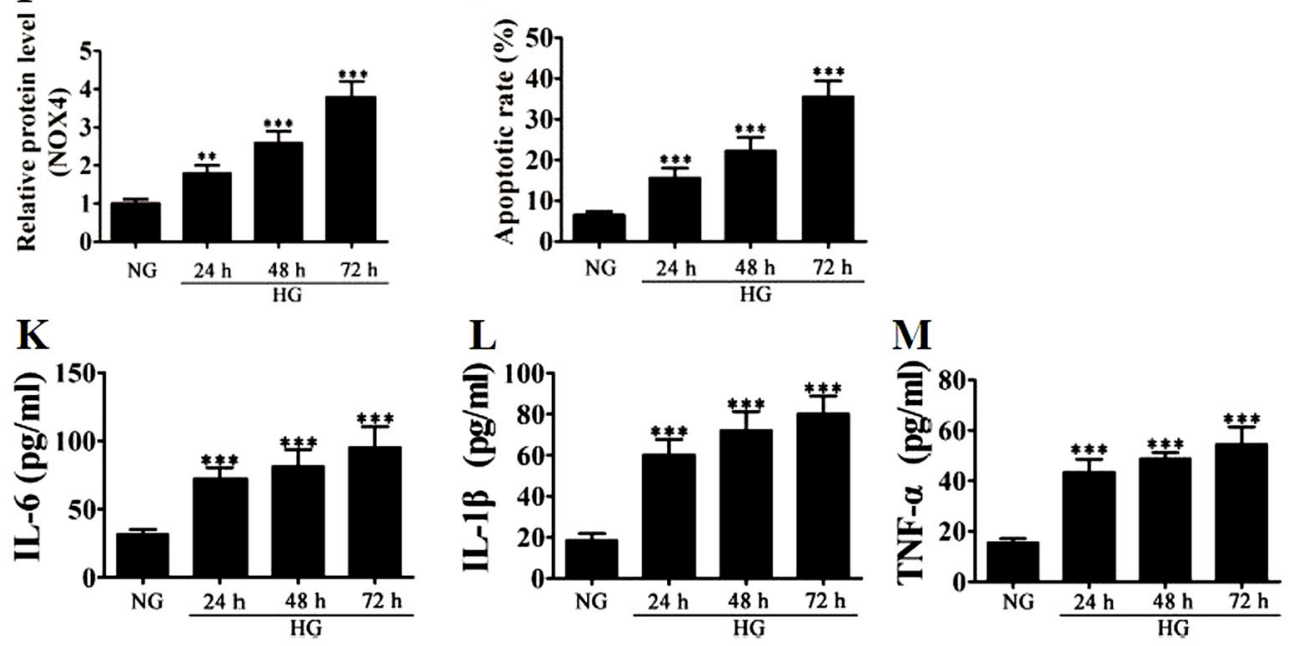

Fig. 3 (See legend on next page.) 


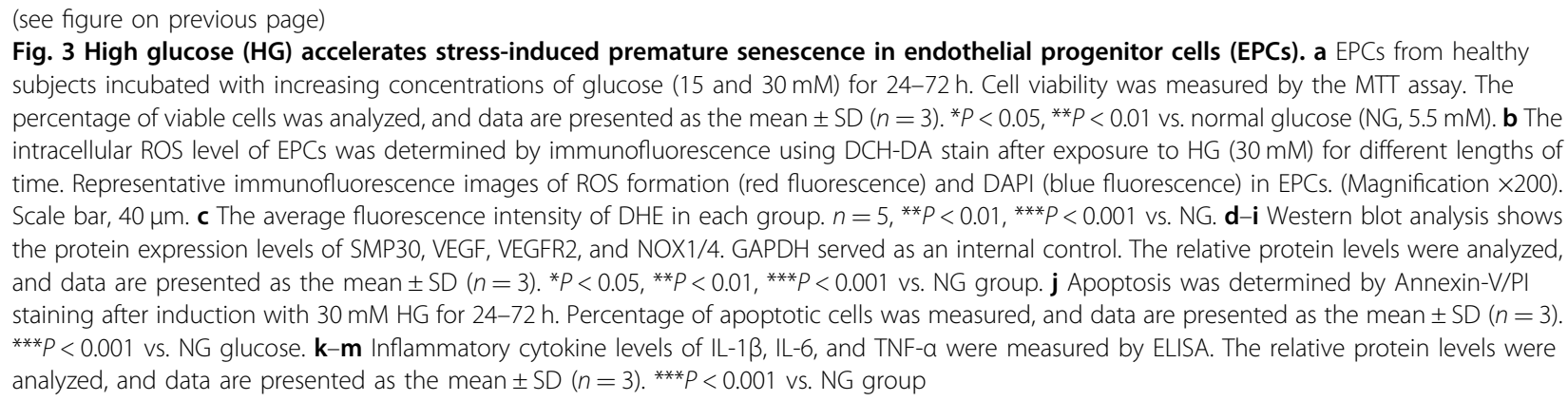

$<100 \mathrm{~nm}$ based on dynamic light scattering (Fig. 5b). Expression of the exosome markers CD4, CD63, and TSG101 was confirmed in the exosome lysis fraction, whereas no markers were observed in the cell lysate (Fig. 5c). Figure $5 \mathrm{~d}$ shows representative micrographs of EPCs containing positively stained Dil-labeled exosomes and the absence of staining in EPCs when exosomes were denatured by boiling.

These results indicate that high glucose-induced stress in EPCs is alleviated by the presence of ADSCs. However, when exosomes are inhibited, the ADSCs have little effect, which suggests that exosomes could be involved in the decrease of EPC senescence under hyperglycemic conditions.

\section{Exosomes derived from Nrf2 overexpressing ADSCs inhibit ROS and inflammatory cytokine expression}

We next assessed whether the protective effects of ADSCs could be increased by the overexpression of Nrf2. Details of the Nrf2 construction and sequence can be found in supplementary information (Supplemental S2). Then, we isolated the exosomes from ADSCs or Nrf2 overexpression ADSCs. Western detection showed that expression of Nrf2 in exosomes from Nrf2 overexpression ADSCs increased compared with that in exosomes that originated from wild-type ADSCs (Fig. 6a,b). Proliferation of EPCs was analyzed by the MTT assay with different concentrations of exosomes from ADSCs or ADSCs overexpressing Nrf2 after exposure to high glucose (30 $\mathrm{mM}$ ) for $48 \mathrm{~h}$ (Fig. 6c). The cell viability in EPCs increased significantly when cultured with ADSCs and exosomes. Furthermore, as quantities of exosomes increased, the increase was elevated. The highest level of viability occurred when ADSCs overexpressing Nrf2 were cultured with EPCs at the highest concentration $(50 \mu \mathrm{g} / \mathrm{ml})$. Tube formation also increased when EPCs were cultured with exosomes from ADSCs overexpressing Nrf2 (Fig. 6d). We evaluated the protein expression levels of SMP30, VEGF, VEGFR, and NOX1/4 after stimulation with ADSC exosomes or Nrf2-ADSC exosomes for $12 \mathrm{~h}$ in high glucose
(Fig. 6e). The exosomes derived from ADSCs overexpressing Nrf2 improved levels of SMP30 and VEGF and increased VEGFR2 phosphorylation, whereas levels of NOX1 and NOX4 were significantly reduced (Fig. $6 \mathrm{f}-\mathrm{j} ; P$ $<0.001$ vs. normal glucose). A similar pattern occurred with the inflammatory cytokine levels of IL-1 $\beta$, IL-6, and TNF- $\alpha$, which were all further reduced by the overexpression of Nrf2 (Fig. 6k-m).

These results indicate that overexpression of Nrf2 accentuates the role of ADSC exosomes in preventing the glucose-induced senescence of EPCs, possibly by inhibiting ROS and inflammatory cytokine expression.

\section{Cutaneous wound healing is accelerated by EPCs combined with exosomes derived from Nrf2 overexpressing ADSCs}

We next compared the effects of the EPCs combined with or without exosome treatments in vitro using a diabetic rat model. After 14 days, full-thickness skin defects in the rats had a significantly reduced ulcerated area when treated with EPCs combined with exosomes, particularly with EPCs combined with exosomes from ADSCs overexpressing Nrf2. Cutaneous wound healing also improved after treatment with EPCs compared with that of an untreated control (Fig. 7a). Granulation tissues in the different treatment groups were measured in $\mathrm{H} \& \mathrm{E}$ stained sections, whereas Masson Trichrome staining was used to assess collagen deposition and tissue fibrosis (Fig. 7b,c). Intracellular ROS levels were determined by immunofluorescence using DCH-DA stain (Fig. 7d). Increased granulation tissue formation, angiogenesis, increased levels of growth factor expression and reduced levels of inflammation and oxidative stress-related proteins were detected in wound beds of rats treated with EPCs combined with exosomes derived from ADSCs and ADSCs overexpressing Nrf2. Immunofluorescence showing microvascular formation confirmed that relative blood vessel density improved following treatment with EPCs combined with exosomes derived from ADSCs and with the highest improvement found in rats treated with EPCs 
A
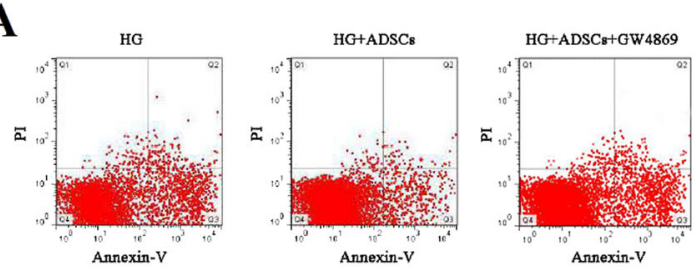

B

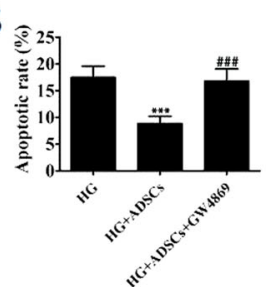

C

HG
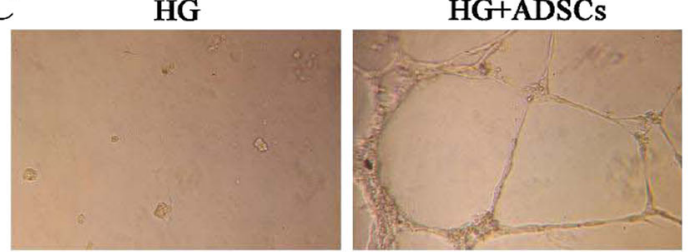

HG+ADSCs+GW4869

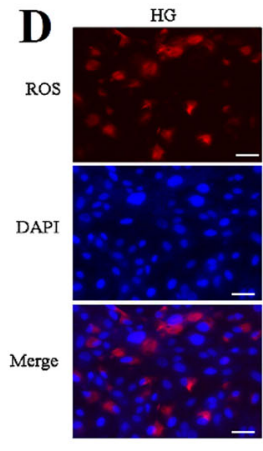

HG+ADSCs

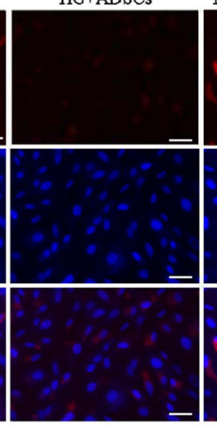

$\mathbf{F}$

$$
\text { S }
$$

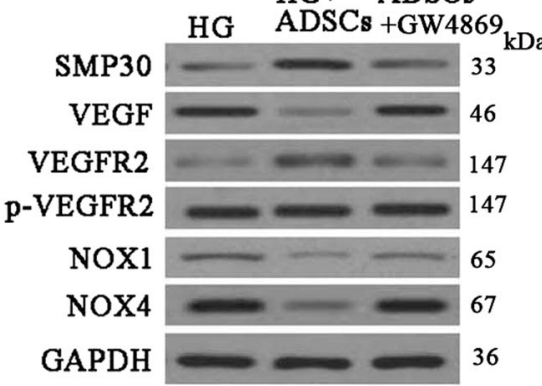

$\mathbf{G}^{-}$

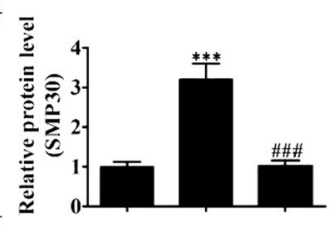

$\mathrm{H}$
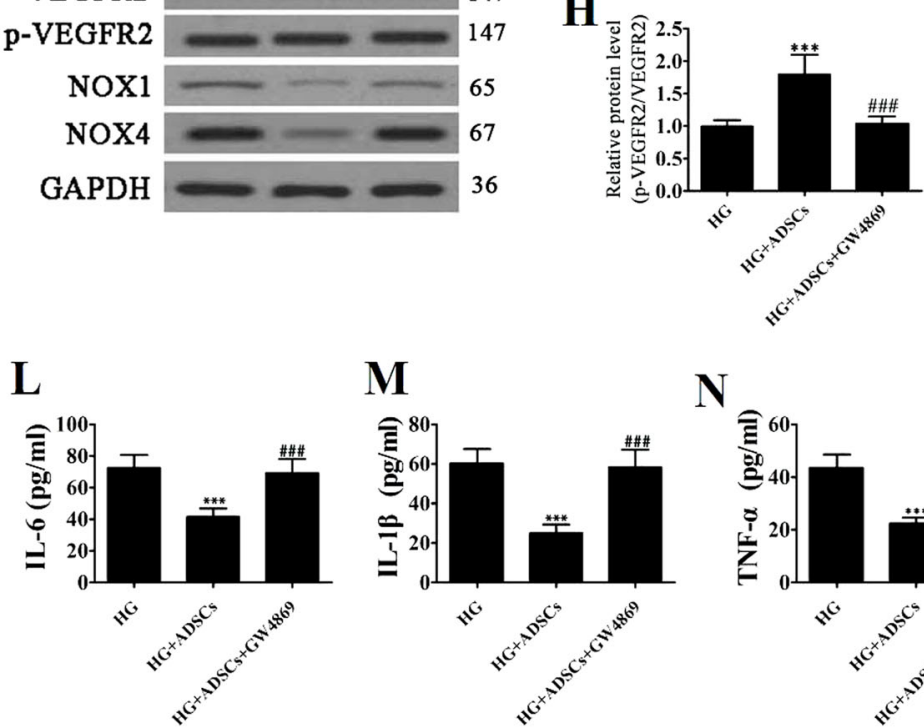

$\mathbf{N}$

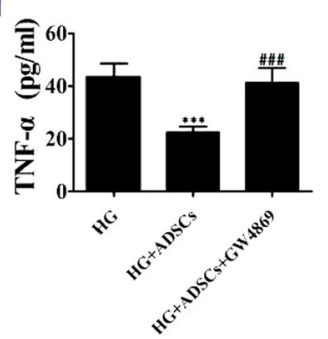

Fig. 4 (See legend on next page.) 
(see figure on previous page)

Fig. 4 Exosomes derived from adipose-derived stem cells (ADSCs) decreased endothelial progenitor cell (EPC) senescence under high glucose (HG) conditions. a The apoptosis rate of EPCs was determined by Annexin-V/PI staining after $24 \mathrm{~h}$ induction with $\mathrm{HG}(30 \mathrm{mM})$ with or without GW4869 $(2.5 \mu \mathrm{M})$ pretreatment for $8 \mathrm{~h}$. b The percentage of apoptotic cells was measured, and data are presented as the mean \pm SD $(n=3)$. ${ }^{* * *} P<0.001$ vs. HG; ${ }^{\# \#} P<0.001$ vs. HG + ADSCs. c The tube formation capability increased in EPCs stimulated with ADSCs, but the promoted effects decreased after pretreatment with GW4869 $(2.5 \mu \mathrm{M})$ for $8 \mathrm{~h}$. $\mathbf{d}$ The intracellular ROS levels were determined by immunofluorescence using DCH-DA

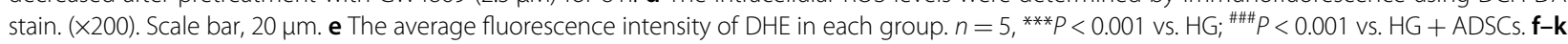
Western blot analysis shows the protein expression levels of SMP30, VEGF, VEGFR2, NOX1, and NOX4. GAPDH served as an internal control. The relative protein level was analyzed, and data are presented as the mean \pm SD $(n=3)$. ${ }^{* * *} P<0.001$ vs. HG; \#\#\# $P<0.001$ vs. HG + ADSCs. I-n Inflammatory cytokine levels of IL-1 $\beta, I L-6$, and TNF-a were measured by ELISA. The relative protein levels were analyzed, and data are presented as

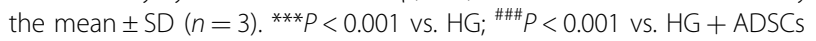
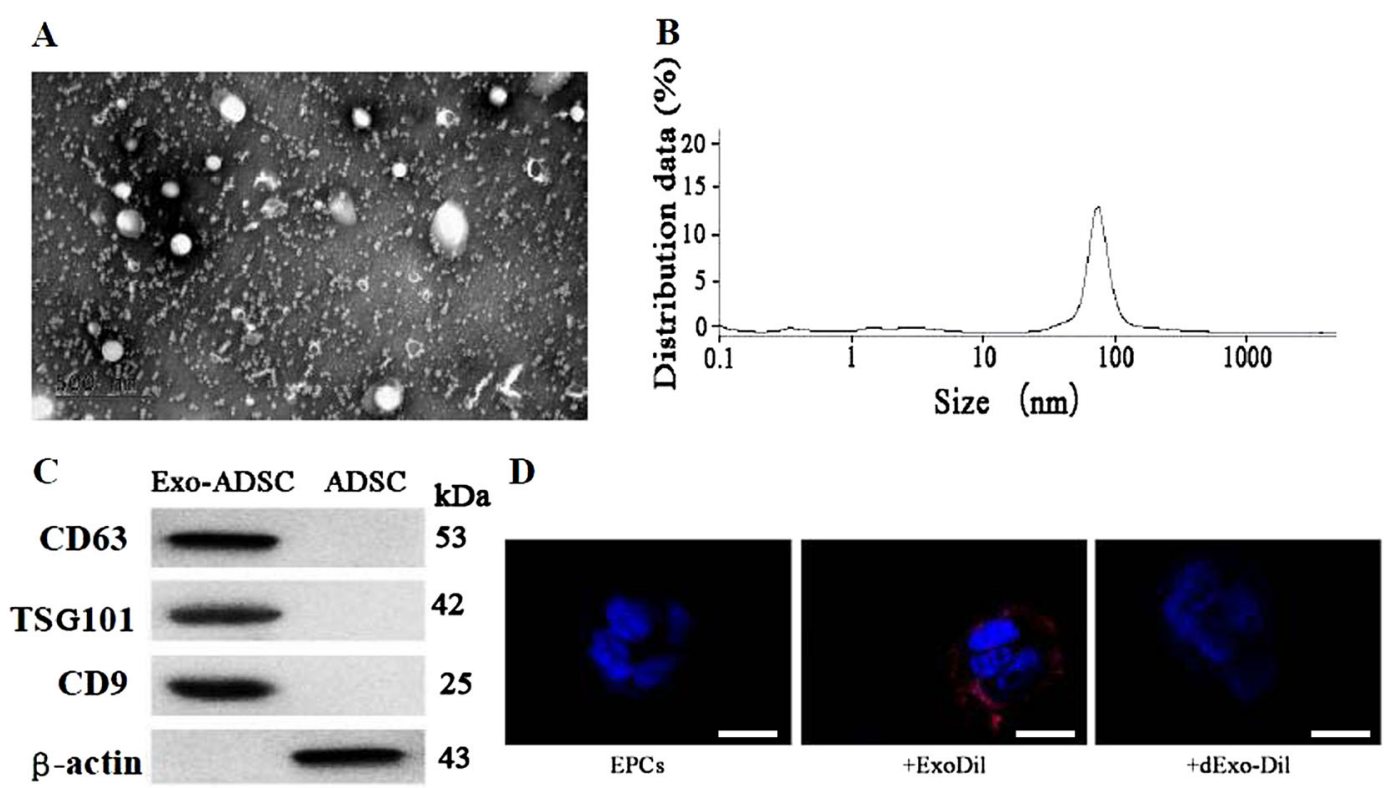

Fig. 5 Isolation and identification of exosomes from adipose-derived stem cell (ADSC) culture medium. a The ultrastructure of ADSC-derived exosomes by transmission electron microscopy. Scale bar, $100 \mathrm{~nm}$. b Size distribution of Exo-ADSCs determined by dynamic light scattering. $\mathbf{c}$ Expression of the exosome markers CD4, CD63, and TSG101 and $\beta$-actin confirmed by immunoblotting. Exosome lysate was loaded into the left lane and cell lysate into the right. $\mathbf{d}$ Representative micrographs of EPCs or EPCs incubated with Dil-labeled Exo (+ExoDil) or with Dil-labeled EVs denatured by boiling (+dExo-Dil). Scale bar, $10 \mu \mathrm{m}$

combined with exosomes from ADSCs overexpressing Nrf2 (Fig. 7e).

Our results demonstrate that exosomes could possibly be used to alleviate the progression of DFUs in patients with diabetes by preventing the senescence of EPCs and inhibiting ROS and inflammatory cytokine expression to reduce inflammation, which would assist wound healing through improved vascularization as observed in our diabetic rat model.

\section{Discussion}

The impaired function and senescence of EPCs in hyperglycemic conditions contribute to increased ROS and impede the healing of DFUs, which are largely caused by peripheral neuropathy associated with $\mathrm{DM}^{4,28}$. We assessed physiological characteristics in patients with diabetes and found significantly elevated levels of fasting glucose, whole blood glycated hemoglobin, VEGF and the inflammatory factors NOX1 and NOX4. By contrast, SMP30 and VEGFR2 were significantly reduced. In this study, we measured the expression levels of SMP30 and phosphorylation of VEGFR2 in EPCs from patients with diabetes and under conditions of high glucose. Stimulation of VEGFR2 by tyrosine kinase activity, in turn, activates endothelial nitric oxide synthase to increase the release of nitric oxide and to extend and increase the blood vessel permeability that is required for angiogenesis $^{29}$. We found that levels of SMP30 and VEGFR2 phosphorylation were significantly reduced in response to high glucose, whereas intracellular ROS and inflammatory 

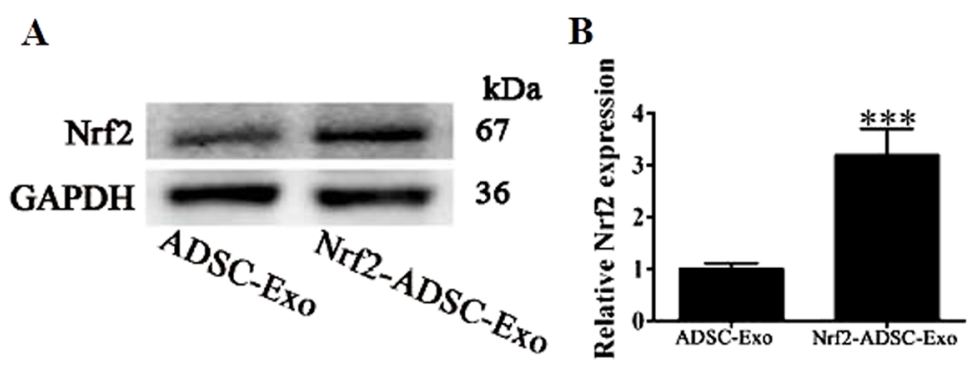

C

D

Control

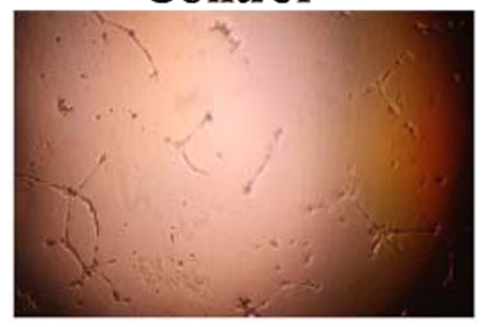

ADSC-Exo
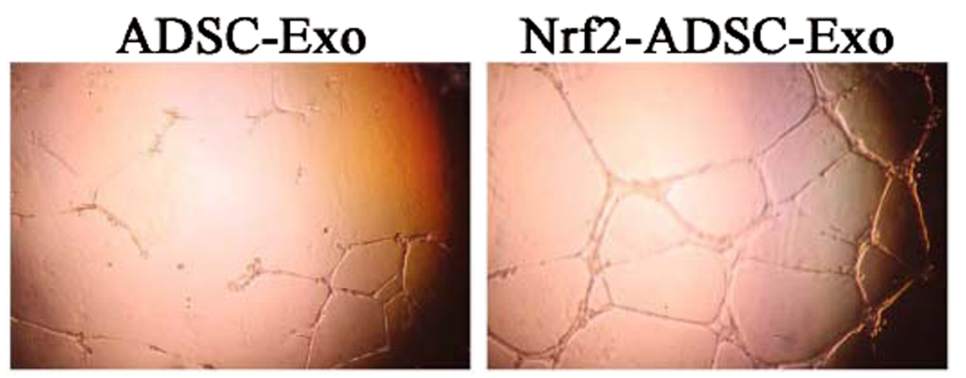

E
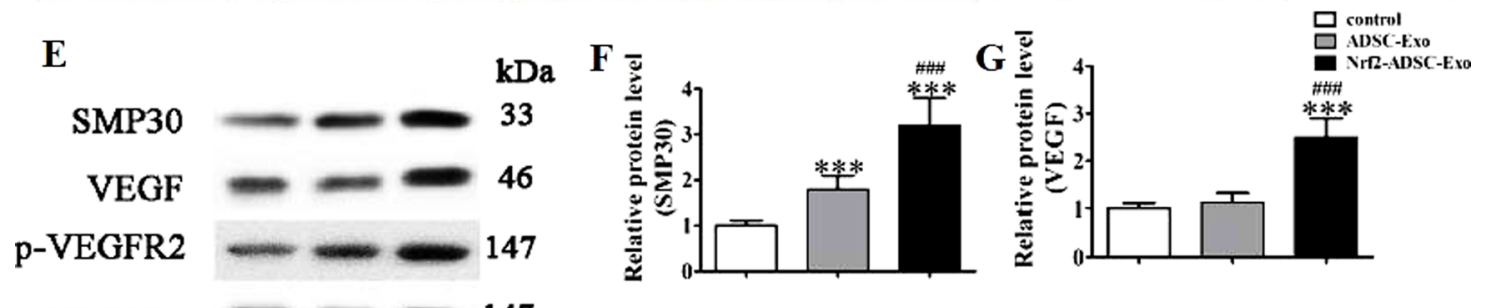

VEGFR2 -147

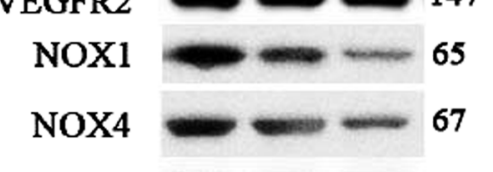

GAPDH
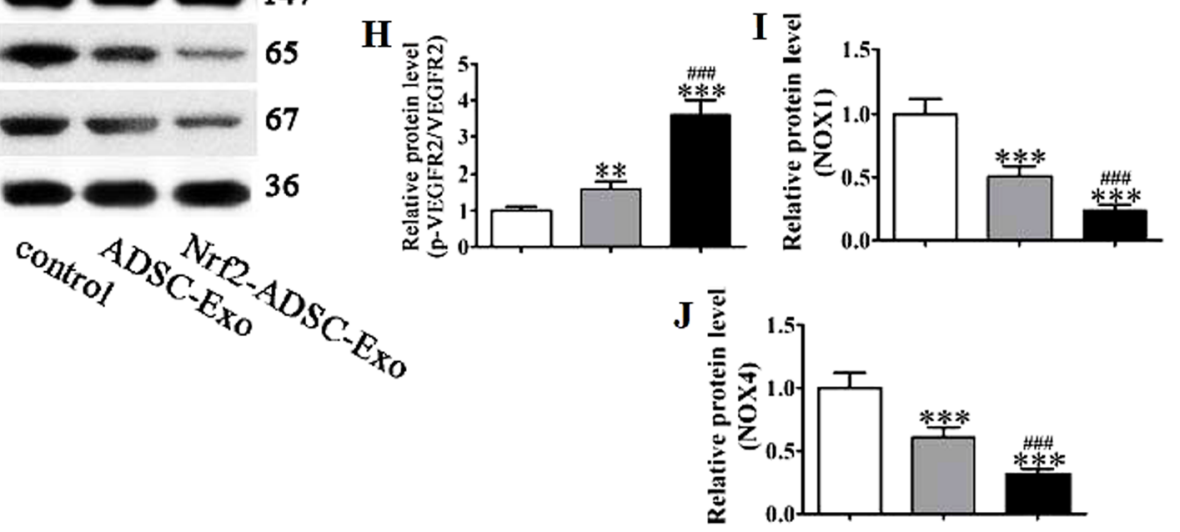

K

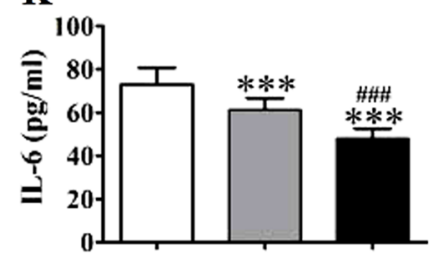

L

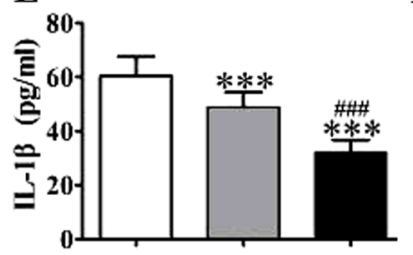

M

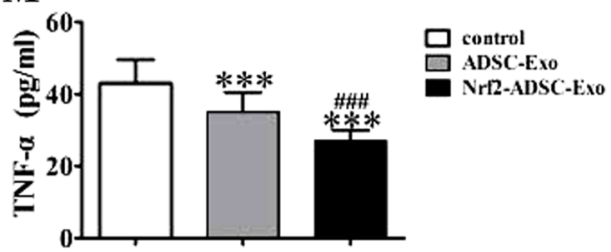

Fig. 6 Exosomes (Exos) derived from adipose-derived stem cells (ADSCs) overexpressing Nrf2 prevent high glucose (HG)-induced senescence by inhibiting ROS and inflammatory cytokine expression. $\mathbf{a}, \mathbf{b}$ The level of Nrf2 in exosomes secreted from ADSCS with or without Nrf2 overexpression. c Proliferation of EPC cells was analyzed by the MTT assay with different concentrations of Exos from ADSCS (ADSC-Exo) or Nrf2 overexpression ADSCs (Nrf2-ADSC-Exo) after exposure to high glucose ( $\mathrm{HG}, 30 \mathrm{mM}$ ) for $48 \mathrm{~h}$. $\mathbf{d}$ Tube formation capability detected in endothelial progenitor cells (EPCs) stimulated with $50 \mathrm{mg} / \mathrm{ml}$ ADSC-Exo or Nrf2-ADSC-Exo for $12 \mathrm{~h}$ under HG $(30 \mathrm{mM})$ condition. e-j Western blot analysis and protein expression levels of SMP30, VEGF, VEGFR, and NOX1/4 after stimulation with ADSC-Exo or Nrf2-ADSC-Exo for $12 \mathrm{~h}$ under HG (30 mM) condition. GAPDH served as an internal control. The relative protein levels were analyzed, and data are presented as the mean $\pm \operatorname{SD}(n=3)$. ${ }^{* * *} P<$ 0.001 vs. normal group. $\mathbf{k}-\mathbf{m}$ Inflammatory cytokine levels of IL-1 $\beta$, IL-6, and TNF-a were measured by ELISA. The relative protein levels were analyzed, and data are presented as the mean $\pm \mathrm{SD}(n=3) .{ }^{*} P<0.05$, ${ }^{* *} P<0.01,{ }^{* * *} P<0.001 \mathrm{vs}$. NG group 


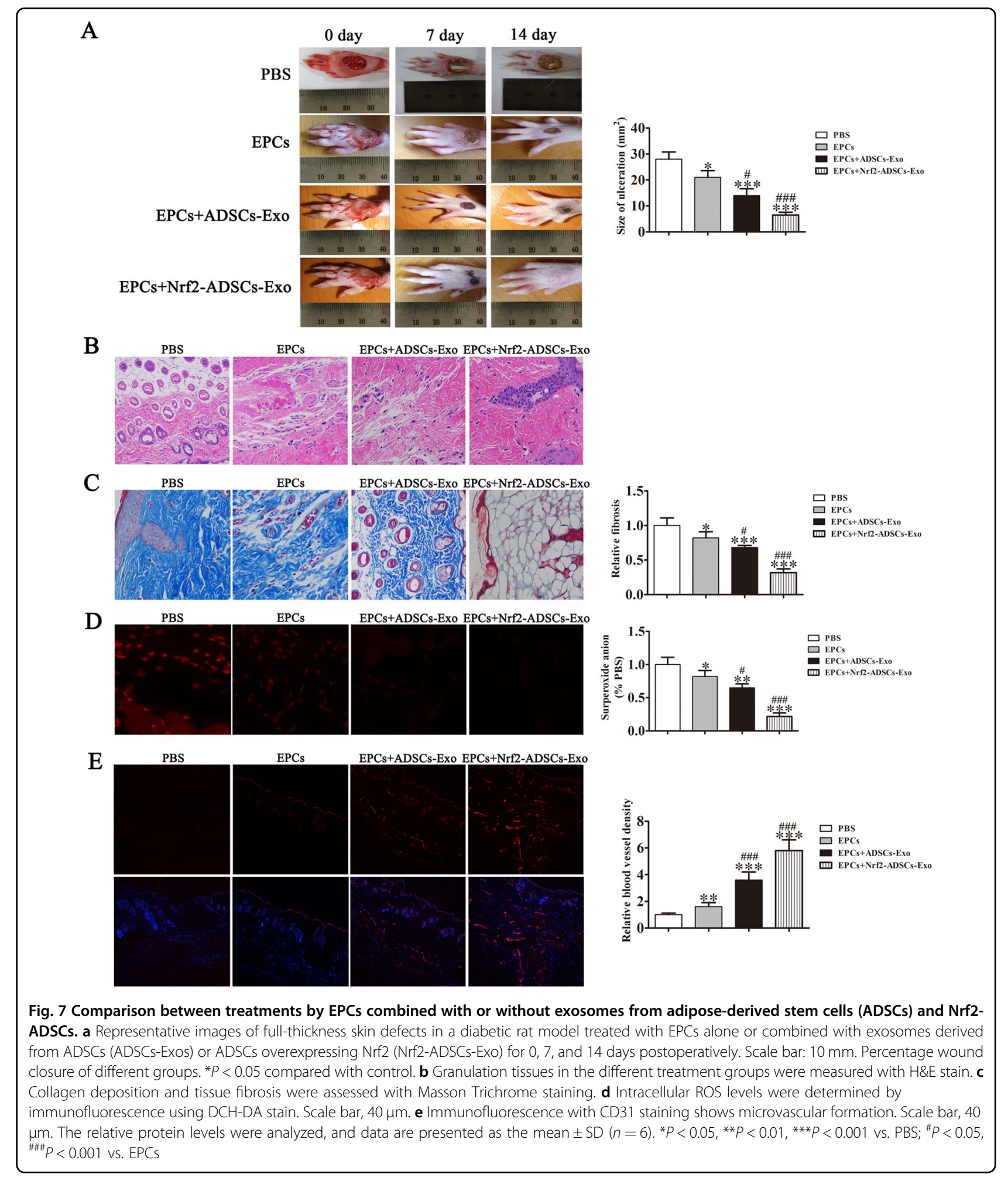

cytokine levels significantly increased. Our results indicate that senescence and oxidative stress increased in patients with DM and are consistent with similar findings in other studies that hyperglycemia promotes the premature senescence of EPCs and increases levels of ROS and inflammation ${ }^{11,30-32}$. We also found that the tube formation capability of EPCs from patients with DM decreased. 
Exosomes from human umbilical cord blood-derived EPCs accelerate wound healing in streptozotocin-induced diabetic rats by promoting angiogenesis through Erk1/ 2 signaling $^{33}$. Similarly, exosomes derived from plateletrich plasma in a diabetic rat model promote reepithelization of chronic cutaneous wounds through YAP activation, a protein involved in maintaining the survival of senescent cells ${ }^{34,35}$. Recent clinical trials using platelet-rich plasma in the treatment of DFUs report promising results $^{36}$. Furthermore, rats with DFUs have been successfully treated with dressings incorporating exosomes from synovium mesenchymal stem cells overexpressing miR-126-3p ${ }^{37}$. We evaluated the therapeutic effects of exosomes secreted from ADSCs on stress-mediated senescence of EPCs induced by high glucose. Apoptosis, glucose-induced ROS levels, and inflammatory cytokine levels were reduced and tube formation capability increased when EPCs were cocultured with ADSCs, but no difference was observed with the addition of the exosome inhibitor GW4869. The protein expression levels of SMP30 and VEGFR2 phosphorylation increased, whereas levels of NOX1 and NOX4 were reduced in EPCs cocultured with ADSCs but not when ADSCs were pretreated with GW4869. Our results indicate that high glucose-induced stress in EPCs is alleviated by the presence of ADSCs, but when exosomes are inhibited, the ADSCs have little effect, suggesting that exosomes could be involved in the decrease of EPC senescence under hyperglycemic conditions.

Nrf2 regulates multiple adaptive responses to oxidative stress and is also involved in cell migration, proliferation, apoptosis, and differentiation ${ }^{16,38}$. Furthermore, Nrf2 has therapeutic effects in rat models of diabetic nephropathy ${ }^{39,40}$. We assessed whether the protective effects of ADSCs could be increased by the overexpression of Nrf2. A significant increase in cell viability occurred in EPCs when cultured with ADSC-derived exosomes, and viability further increased when Nrf2 was overexpressed. Tube formation also increased when EPCs were cultured with exosomes from ADSCs overexpressing Nrf2. Exosomes derived from ADSCs overexpressing Nrf2 improved levels of SMP30 and VEGF and increased VEGFR2 phosphorylation, whereas levels of ROS and inflammatory cytokine levels were significantly reduced. These results indicate that overexpression of Nrf2 in ADSC exosomes further prevents the glucose-induced senescence of EPCs, possibly by inhibiting ROS and inflammatory cytokine expression.

We found that ADSCs secreting exosomes promoted proliferation and angiopoiesis in EPCs in a high glucose environment and that overexpression of Nrf2 increased this protective effect. The use of exosomes from ADSCs overexpressing Nrf2 in a diabetic rat model significantly reduced the ulcerated area. Wound healing also improved after treatment with ADSCs compared with that in an untreated control, with increased granulation tissue formation, angiogenesis, increased levels of growth factor expression and reduced levels of inflammation and oxidative stress-related proteins detected in wound beds of rats treated with exosomes derived from ADSCs overexpressing Nrf2. Relative blood vessel density also improved following treatment with the exosomes.

To conclude, our results demonstrate that exosomes could possibly be used to alleviate the progression of DFUs in patients with diabetes by preventing senescence of EPCs and inhibiting ROS and inflammatory cytokine expression to reduce inflammation and thereby assist wound healing by improved vascularization as observed in our streptozotocin-induced diabetic rat model. Our data suggest that exosomes from ADSCs can potentially promote wound healing, particularly when overexpressing Nrf2 and therefore that transplantation of exosomes may be suitable for clinical application in the treatment of DFUs.

\section{Acknowledgements \\ The National Natural Science Foundation of China (Grant no. 81671793) supported this study. \\ Author details \\ ${ }^{1}$ Department of Interventional \& Vascular Surgery, Tenth People's Hospital of Tongji University, No. 301 Middle Yan Chang Road, Shanghai 200072, China. ${ }^{2}$ Institute of Interventional \& Vascular Surgery, Tongji University, No. 301 Middle Yan Chang Road, Shanghai 200072, China. ${ }^{3}$ National United Engineering Laboratory for Biomedical Material Modification, Branden Biomedical Park, Qihe Advanced Science \& High Technology Development Zone, Qihe, Shandong 251100, China. 'Zhuhai people's hospital, No. 79 Kangning Road, Xiangzhou District, Zhuhai City 519000, China}

\section{Author contributions}

X.L., H.Z., W.L., R.S. and S.H. generated and analyzed data; L.L., X.X., and M.L. designed experiments and drafted the manuscript. All authors approved the version of the manuscript to be published.

Conflict of interest

The authors declare that they have no conflict of interest.

\section{Publisher's note}

Springer Nature remains neutral with regard to jurisdictional claims in published maps and institutional affiliations.

Supplementary information accompanies this paper at https://doi.org/ 10.1038/s12276-018-0058-5.

Received: 24 July 2017 Revised: 10 October 2017 Accepted: 19 October 2017.

Published online: 13 April 2018

\footnotetext{
References

1. Ramsey, S. D. et al. Incidence, outcomes, and cost of foot ulcers in patients with diabetes. Diabetes Care 22, 382-387 (1999).

2. Han, G. \& Ceilley, R. Chronic wound healing: a review of current management and treatments. Adv. Ther. 34, 599-610 (2017).

3. Boulton, A. J., Vileikyte, L., Ragnarson-Tennvall, G. \& Apelqvist, J. The global burden of diabetic foot disease. Lancet 366, 1719-1724 (2005).

4. Fetterolf, D. E., Istwan, N. B. \& Stanziano, G. J. An evaluation of healing metrics associated with commonly used advanced wound care products for the treatment of chronic diabetic foot ulcers. Manag. Care. 23, 31-38 (2014).
} 
5. Kavitha, K. V. et al. Choice of wound care in diabetic foot ulcer: a practical approach. World J. Diabetes 5, 546-556 (2014).

6. Trieb, K. The Charcot foot: pathophysiology, diagnosis and classification. Bone Joint J. 98-B, 1155-1159 (2016).

7. Hwang, D. J. et al. Association between diabetic foot ulcer and diabetic retinopathy. PLOS ONE 12, e0175270 (2017).

8. Bowering, C. K. Diabetic foot ulcers. Pathophysiology, assessment, and therapy. Can. Fam. Physician 47, 1007-1016 (2001).

9. Kota, S. K., Meher, L. K., Jammula, S., Krishna, S. V. \& Modi, K. D. Aberrant angiogenesis: the gateway to diabetic complications. Indian J. Endocrinol. Metab. 16, 918-930 (2012).

10. Brem, $\mathrm{H}$. et al. Wound-healing protocols for diabetic foot and pressure ulcers. Surg. Technol. Int. 11, 85-92 (2003).

11. Issan, Y. et al. Endothelial progenitor cell function inversely correlates with long-term glucose control in diabetic patients: association with the attenuation of the heme oxygenase-adiponectin axis. Can. J. Cardiol. 28, 728-736 (2012).

12. Zubiri, I. et al. Kidney tissue proteomics reveals regucalcin downregulation in response to diabetic nephropathy with reflection in urinary exosomes. Transl. Res. 166, 474-484 (2015). e474.

13. Arun, P., Aleti, V., Parikh, K., Manne, V. \& Chilukuri, N. Senescence marker protein 30 (SMP30) expression in eukaryotic cells: existence of multiple species and membrane localization. PLOS ONE 6, e16545 (2011).

14. Yamauchi, $H$. et al. Senescence marker protein-30 deficiency impairs angiogenesis under ischemia. Free Radic. Biol. Med. 94, 66-73 (2016).

15. Long, M. et al. An essential role of NRF2 in diabetic wound healing. Diabetes 65, 780-793 (2016).

16. Kensler, T. W., Wakabayashi, N. \& Biswal, S. Cell survival responses to environmental stresses via the Keap1-Nrf2-ARE pathway. Annu. Rev. Pharmacol. Toxicol. 47, 89-116 (2007).

17. Nguyen, T., Nioi, P. \& Pickett, C. B. The Nrf2-antioxidant response element signaling pathway and its activation by oxidative stress. J. Biol. Chem. $\mathbf{2 8 4}$ 13291-13295 (2009).

18. Kolluru, G. K., Bir, S. C. \& Kevil, C. G. Endothelial dysfunction and diabetes: effects on angiogenesis, vascular remodeling, and wound healing. Int. J. Vasc. Med. 2012, 918267 (2012).

19. $\mathrm{Xu}, \mathrm{X}$. et al. Genetic variants of nuclear factor erythroid-derived 2-like 2 associated with the complications in Han descents with type 2 diabetes mellitus of Northeast China. J. Cell. Mol. Med. 20, 2078-2088 (2016).

20. Massee, M. et al. Type I and II diabetic adipose-derived stem cells respond in vitro to dehydrated human amnion/chorion membrane allograft treatment by increasing proliferation, migration, and altering cytokine secretion. Adv. Wound Care 5, 43-54 (2016).

21. Garcia-Contreras, M., Vera-Donoso, C. D., Hernandez-Andreu, J. M., GarciaVerdugo, J. M. \& Oltra, E. Therapeutic potential of human adipose-derived stem cells (ADSCs) from cancer patients: a pilot study. PLOS ONE 9, e113288 (2014).

22. Yuan, Y. et al. Mesenchymal stem cell-conditioned media ameliorate diabetic endothelial dysfunction by improving mitochondrial bioenergetics via the Sirt1/AMPK/PGC-1alpha pathway. Clin. Sci. 130, 2181-2198 (2016).
23. $\mathrm{Hu}$, L. et al. Exosomes derived from human adipose mensenchymal stem cells accelerates cutaneous wound healing via optimizing the characteristics of fibroblasts. Sci. Rep. 6, 32993 (2016).

24. Wu, K. et al. The effects of microvesicles on endothelial progenitor cells are compromised in type 2 diabetic patients via downregulation of the miR-126/ VEGFR2 pathway. Am. J. Physiol. Endocrinol. Metab. 310, E828-837 (2016).

25. Meng, S. et al. Downregulation of microRNA-130a contributes to endothelial progenitor cell dysfunction in diabetic patients via its target Runx3. PLOS ONE 8, e68611 (2013).

26. Zhao, L. et al. Exosomes derived from human pulmonary artery endothelial cells shift the balance between proliferation and apoptosis of smooth muscle cells. Cardiology 137, 43-53 (2017).

27. Shi, R. et al. Localization of human adipose-derived stem cells and their effect in repair of diabetic foot ulcers in rats. Stem Cell Res. Ther. 7, 155 (2016).

28. Edmonds, M. E., Foster, A. V. \& McColgan, M. 'Dermagraft': a new treatment for diabetic foot ulcers. Diabet. Med. 14, 1010-1011 (1997).

29. Ruszkowska-Ciastek, B. et al. A preliminary evaluation of VEGF-A, VEGFR1 and VEGFR2 in patients with well-controlled type 2 diabetes mellitus. J. Zhejiang Univ. Sci. B 15, 575-581 (2014).

30. Issan, Y. et al. Elevated level of pro-inflammatory eicosanoids and EPC dysfunction in diabetic patients with cardiac ischemia. Prostaglandins Other Lipid Mediat. 100-101, 15-21 (2013).

31. Zhou, L. et al. High glucose induces renal tubular epithelial injury via Sirt1/NFkappaB/microR-29/Keap1 signal pathway. J. Transl. Med. 13, 352 (2015).

32. Fu, C., Li, B., Sun, Y., Ma, G. \& Yao, Y. Bradykinin inhibits oxidative stress-induced senescence of endothelial progenitor cells through the B2R/AKT/RB and B2R/ EGFR/RB signal pathways. Oncotarget 6, 24675-24689 (2015).

33. Zhang, J. et al. Exosomes derived from human endothelial progenitor cells accelerate cutaneous wound healing by promoting angiogenesis through Erk1/2 signaling. Int. J. Biol. Sci. 12, 1472-1487 (2016).

34. Guo, S. C. et al. Exosomes derived from platelet-rich plasma promote the reepithelization of chronic cutaneous wounds via activation of YAP in a diabetic rat model. Theranostics 7, 81-96 (2017).

35. Ma, K. et al. Nuclear accumulation of Yes-Associated Protein (YAP) maintains the survival of doxorubicin-induced senescent cells by promoting survivin expression. Cancer Lett. 375, 84-91 (2016).

36. Suthar, M., Gupta, S., Bukhari, S. \& Ponemone, V. Treatment of chronic nonhealing ulcers using autologous platelet rich plasma: a case series. J. Biomed. Sci. 24, 16 (2017).

37. Tao, S. C. et al. Chitosan wound dressings incorporating exosomes derived from microRNA-126-overexpressing synovium mesenchymal stem cells provide sustained release of exosomes and heal full-thickness skin defects in a diabetic rat model. Stem Cells Transl. Med. 6, 736-747 (2017).

38. Jang, J., Wang, Y., Kim, H. S., Lalli, M. A. \& Kosik, K. S. Nrf2, a regulator of the proteasome, controls self-renewal and pluripotency in human embryonic stem cells. Stem Cells 32, 2616-2625 (2014).

39. Zheng, $\mathrm{H}$. et al. Therapeutic potential of Nrf2 activators in streptozotocininduced diabetic nephropathy. Diabetes 60, 3055-3066 (2011).

40. Jiang, $\mathrm{T}$. et al. The protective role of Nrf2 in streptozotocin-induced diabetic nephropathy. Diabetes 59, 850-860 (2010). 